\title{
Blind Adaptive Equalization of Complex Signals based on the Constant Modulus Algorithm
}

\author{
D.R. Srinivas \\ ECE Department \\ G. Pulla Reddy Engineering College \\ Andhra Pradesh, India
}

\author{
K.E. Sreenivasa Murthy \\ Principal, SVIT \\ Anantapur \\ Andhra Pradesh, India
}

\begin{abstract}
The paper discuss, applicability of the second-order Newton gradient descent method for blind equalization of complex signals based on the Constant Modulus Algorithm (CMA). The Constant Modulus (CM) loss function is real with complex valued arguments, and, hence, non-analytic. The Hessian for noiseless FIR channels and rederive the known fact that the full Hessian of the CM loss function is always singular in a simpler manner. The channel model shows that the perfectly equalizing solutions are stationary points of the CM loss function and also evaluate the bit error rate. The paper also discuss of the full Newton method. Finally, to validate the proposed algorithm, simulation studies have been carried out and results are presented and compared. The simulation results show the effectiveness of the proposed algorithm.
\end{abstract}

\section{General Terms}

Mobile communications, FIR channels.

\section{Keywords}

Analytic functions, blind equalization, complex Newton method, constant modulus algorithm (CMA).

\section{INTRODUCTION}

For finite length FIR channel models it is common to use equalizers with a tapped delay line structure. The taps of the equalizer are updated using adaptive algorithms, which usually depend on the presence of training symbols. Blind equalizers, originally proposed by Sato [1], do not require training symbols, thus, potentially improving the information spectral efficiency. A well-known candidate among the blind adaptive algorithms is the Constant Modulus algorithm (CMA) proposed by Godard [2] for two-dimensional digital communication systems (M-ary quadrature amplitude modulation (16-QAM) signals), and by Treichler and Agee [3], for blind equalization of pulse-amplitude modulation (PAM) signals. CMA is a preferred choice for blind equalization because of its robustness and its ease of implementation [4]. Convergence analysis of CMA can be found in [5] and [6]. The speed of convergence is very important for practical systems. It is well known that the second-order Newton method has fast convergence. However, the Newton method requires the computation of the Hessian of the cost function for its implementation. Emphasize that it works with complex signals as this makes the problem of understanding second-order CMA algorithms significantly more difficult than the real case. In particular, unlike the real case, there are no isolated local minima of the Constant Modulus (CM) loss function in the complex situation and as a consequence the Hessian of the CM loss function is always singular, which means that the fast converging Newton method can not be used in practice without modification. Theoretically show that this intrinsic singularity prevents one from using the Newton algorithm without precautionary modifications. This is accomplished by using the framework and methodology developed in [8] to analyze the Newton method applied to minimizing the $\mathrm{CM}$ loss function. Some modified versions of the Newton method as applied to constant modulus algorithm can be found in [7]-[8].

1) The full Hessian of the CM loss function is proven to be intrinsically singular. This fact is actually well known [4], [7], but the proof given in this paper is much simpler and is based on the complex Hessian derived using the framework of Wirtinger calculus.

2) It shows that the perfectly equalizing solutions are stationary points of the CM loss function. We compute the form of the Hessian for the noiseless FIR channel model and evaluate it at a perfectly equalizing solution.

3) Conditions are given which ensure that the leading partial Hessian is positive definite and necessarily full rank.

4) It shows that at a perfectly equalizing solution for a noiseless full rank channel, the leading partial Hessian is full rank assuming that the channel input data sequence is sufficiently subGaussian.

5) If the leading partial Hessian is full rank, then the necessarily Singular 2NX2Nfull Hessian is shown to attain the maximal rank of $2 \mathrm{~N}-1$.

6) The given form of the vector which spans the one dimensional null space of a maximal rank full Hessian at a perfectly equalizing solution for a noiseless FIR channel.

7) It presents a novel phase-enforcing regularization of the full Newton algorithm and discuss its implication for developing practical Newton algorithms as applied to the CMA.

\subsection{History}

In the literature, blind equalization algorithms blossomed in the 1980's. The two principal precursors are Lucky's blind decisiondirection algorithm [4] and Sato's algorithm [1]. the CM criterion was introduced for blind equalization of QAM signals in [6] and of pulse-amplitude modulation (PAM) and FM signals in [3]. By the end of the 1980's blind equalizers were commercialized for microwave radio [9]. By the mid 1990's blind equalizers were realized in very large scale integration (VLSI) for high definition television (HDTV) set-top cable demodulators [2]. The current 
explosion of interest in the $\mathrm{CM}$ criterion stems from blind processing applications in emerging wireless communication technology (e.g., blind equalization, blind source separation, and blind antenna steering) and from CMA's record of practical success.

\subsection{Our Mission}

This paper is intended to be a resource to both readers Experienced in blind equalization as well as those new to the subject. In a tutorial style, this is provides background in fractionally spaced equalizer (FSE) modeling and design.(For baud-spaced equalizer (BSE) design, e.g.,[5], [6], [7], and [8].) then illustrates several low-dimensional examples that help to characterize the behavior of FSE's adapted under the constant modulus criterion. It constructs a categorization of literatue focusing on the application of the $\mathrm{CM}$ criterion to blind equalization. This is providing the reader with a valuable tool for further research. Our attempt to be exhaustive is justified only by the relative infancy of the subfield; evidence of the emerging status of this literature is seen in the wealth of conference papers in references.

Following the introductory FSE tutorial, presents a novel view of classical non blind adaptive equalization that illuminates the connection between the MSE and CM criteria. Specifically, the LMS-with-training strategy requires pre selection of a design variable, namely training sequence delay, that may lead to a potentially suboptimal solution. The delay-optimized MSE (a function of equalizer parameters only) yields a cost surface for which a simple LMS-like parameter update algorithm is not known to exist. Remarkably, the CM criterion offers aproxy for this surface for which there exists a (blind) parameter update algorithm, namely, CMA.

\subsection{Fractionally Spaced Linear Equalization}

This section describes the fractionally spaced equalization scenario and presents some fundamental results regarding minimum MSE equalizers. This material is primarily intended to provide background and context. For simplicity, our focus is restricted to a T/2-spaced FSE, where $\mathrm{T}$ denotes the baud, or symbol, duration. All results are extendible to the more genera $\mathrm{T} / 2$-spaced case. Examples of seminal work on fractionally spaced equalization include [4], [6] and [8], while more comprehensive references are [5] and [7].

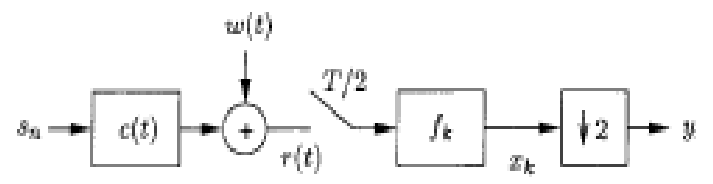

Figure. 1 Baseband model of single-channel communication system with $\mathrm{T} / 2$-spaced receiver.

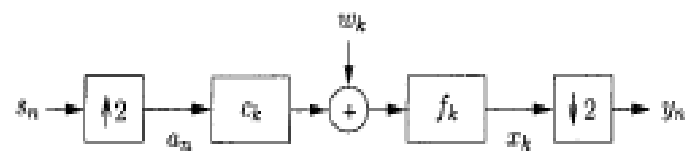

Figure. 2 Multirate system model.

\subsection{Multirate and Multichannel System Models:}

Consider the single-channel model illustrated in Fig.1. A possibly complex-valued) T-spaced symbol sequence $\{\mathrm{Sn}\}$ is transmitted through a pulse shaping filter, modulated onto a propagation channel, and demodulated. Assumption of all processing between the transmitter and receiver is linear and time invariant (LTI) and can thus be described by the continuous-time impulse response $\mathrm{c}(\mathrm{t})$. The received signal $\mathrm{r}(\mathrm{t})$ is also corrupted by additive channel noise, whose baseband equivalent and it denote by $\mathrm{u}(\mathrm{t})$. The received signal is then sampled at $\mathrm{T} / 2$-spaced intervals and filtered by a $\mathrm{T} / 2$-spaced finite impulse response (FIR) equalizer of length 2N. (An even length is chosen for notational simplicity.) This filtering can be regarded as a convolution of the sampled received sequence with the equalizer coefficients $\mathrm{fk}$. Finally, the FSE output is $\{\mathrm{xk}\}$ decimated by a factor of two to create the T-spaced output sequence $\{\mathrm{yn}\}$. Decimation is accomplished by disregarding alternate samples, thus producing the baud-spaced "soft decisions" yn. In general, all quantities are complex valued. For clarity, we reserve the index $n$ for baud-spaced quantities and the index $\mathrm{k}$ for fractionally spaced quantities throughout the paper.

It derives the equivalence between the continuous-time model in Fig. 3 and the discrete time models in Figs. 2 and 3, both constructed using -spaced samples of and . Fig.2 depicts the multirate model while Fig.3 depicts the multichannel model. Though our derivation of the discrete-time models is based on the single-channel system in Fig. 1, the equivalence between the multirate and multichannel models suggests that the model on a two-sensor(T-sampled) communication system instead. For a concise discussion on the equivalence between temporal and spatial diversity, see [4].

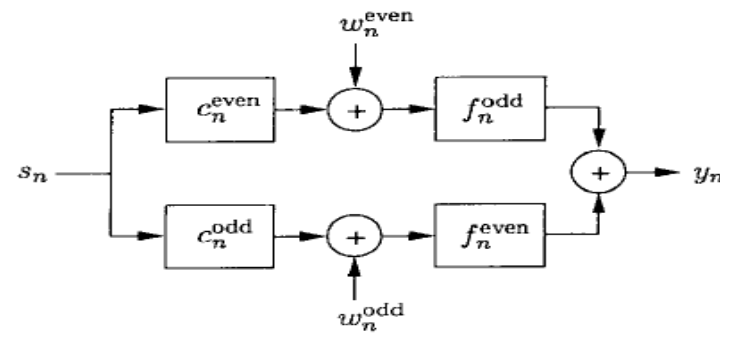

Figure.3 Multichannel system model.

\section{SYSTEM MODEL}

Zero-mean, nonzero bounded, fourth-order moment i.i.d signals $a(n)$ are assumed to be noiselessly transmitted through an unknown linear time invariant channel resulting in a received zero-mean output sequence $x(n)$ which is processed via an $\mathrm{N}$ tap FIR equalizer with weights $\mathrm{W}$. The equalizer output sequence is given

$y(n)=\sum_{k=0}^{N-1} \bar{\omega}(k) x(n-k) \equiv w^{H} X(n)$ 
$l(w)=E\left\{\left(w^{H} X X^{H} w-\rho\right)^{2}\right\}$

\section{STATIONARY POINTS OF CM LOSS}

The condition for $\mathrm{W}$ to be a stationary point of the CM loss function (2) is

$$
(A(w)-\rho B) w=0
$$

An important problem for the above conventional CMA, however, is that it is phase-blind, because the cost function can only deal with the modulus of the equalizer output. As a result, the equalizer output signal constellation suffers from an arbitrary phase rotation at a rate equal to the carrier frequency offset rate. In order to achieve simultaneous blind equalization and carrier phase recovery, the cost function can be rewritten as the following form

$$
J(k)=J_{R}(k)+J_{I}(k)
$$

\section{CONSTANT MODULUS ALGORITHM}

Consider the baseband model of a digital transmission channel characterized by a finite impulse response (FIR) filter and an additive white noise. The received signal can be modeled as

$x(k)=\sum_{i=0}^{L-1} h(i) a(k-i) e^{j \phi(k)}+n(k)$

The equalizer output is thus

$$
y(k)=W^{H}(k) X(k)
$$

\section{RESULTS AND DISCUSSIONS}

To validate the proposed algorithm, numerical simulation studies have been carried out using MATLAB. All simulation experiments described in this section employ a complex equalizer of transversal filter structure with 9 tap weights, unless otherwise mentioned, and the equalizers were initialized with the central tap weight set to 1 and others set to zero. The signal to noise ratio (SNR) at the input of the equalizer is defined as

$$
S N R=10 \log _{10} \frac{E\left\lfloor\left. a(k) * h(k)\right|^{2}\right\rfloor}{\sigma_{n}^{2}}(d B)
$$

The simulation results are shown in from Fig. 4 to Fig.9. The Fig. 4 is an input signal and the Fig. 5 is an modulated signal and the Fig. 6 is an noise signal, Fig.7 is an filter ouput, Fig.8 is an 16QAM demodulated output and finally the Fig. 9 show the comparison of Newton Method-CMA and CMA. From the simulation results, it can be observed that the proposed CMA algorithm gives better results when compared with the NM-CMA algorithm.

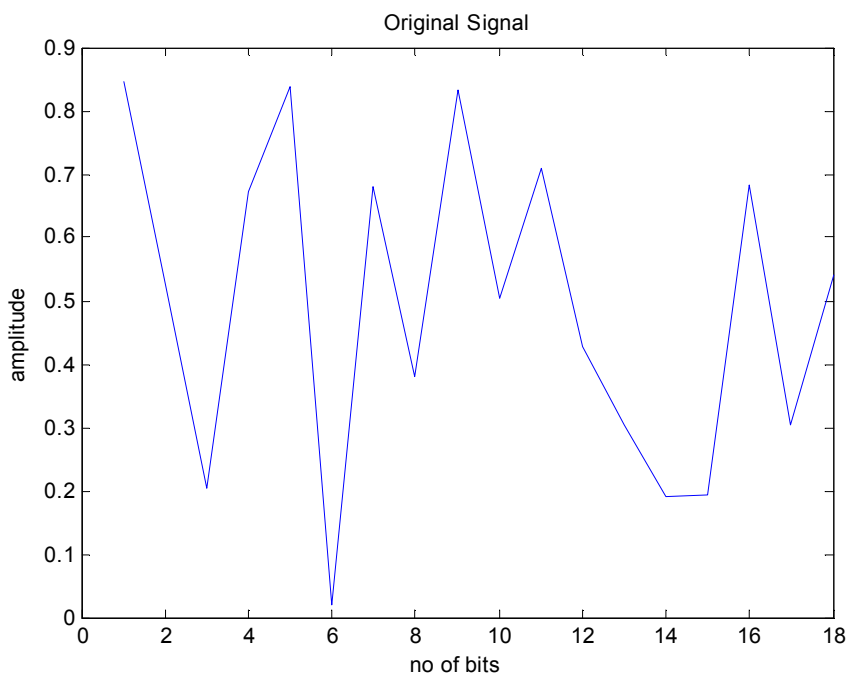

Figure.4 Random sequence of input signal

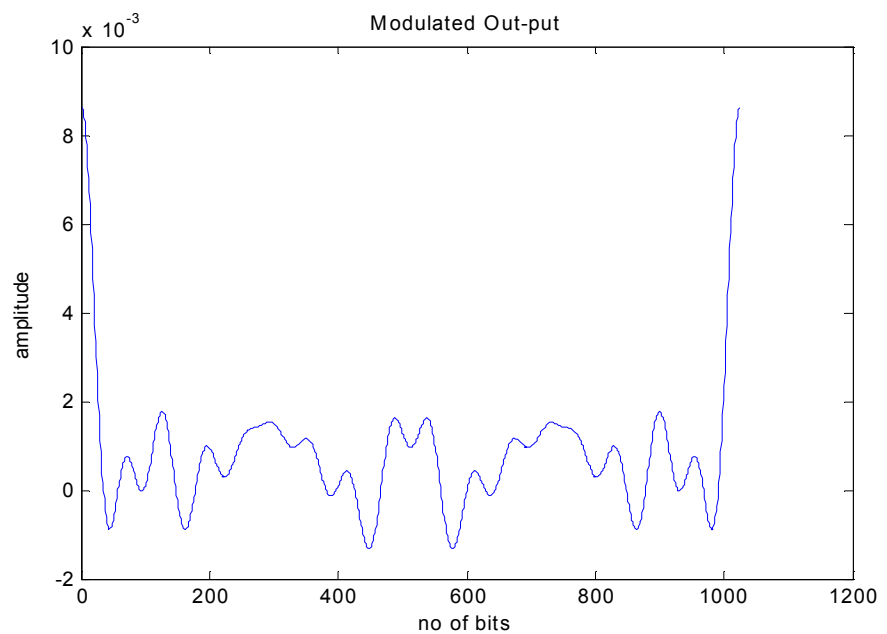

Figure 5 16-QAM Modulated signal

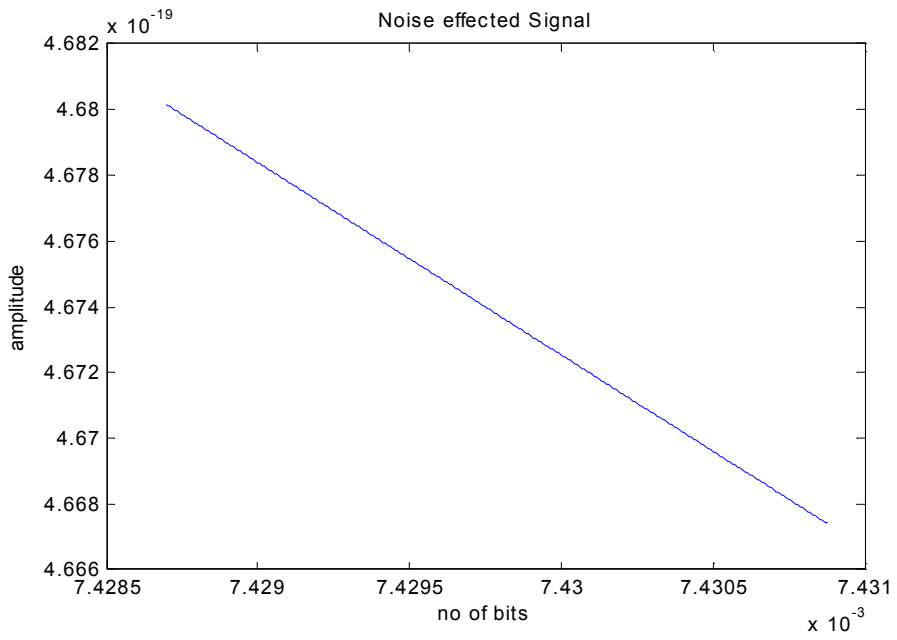

Fig.6 Noise effected signal 

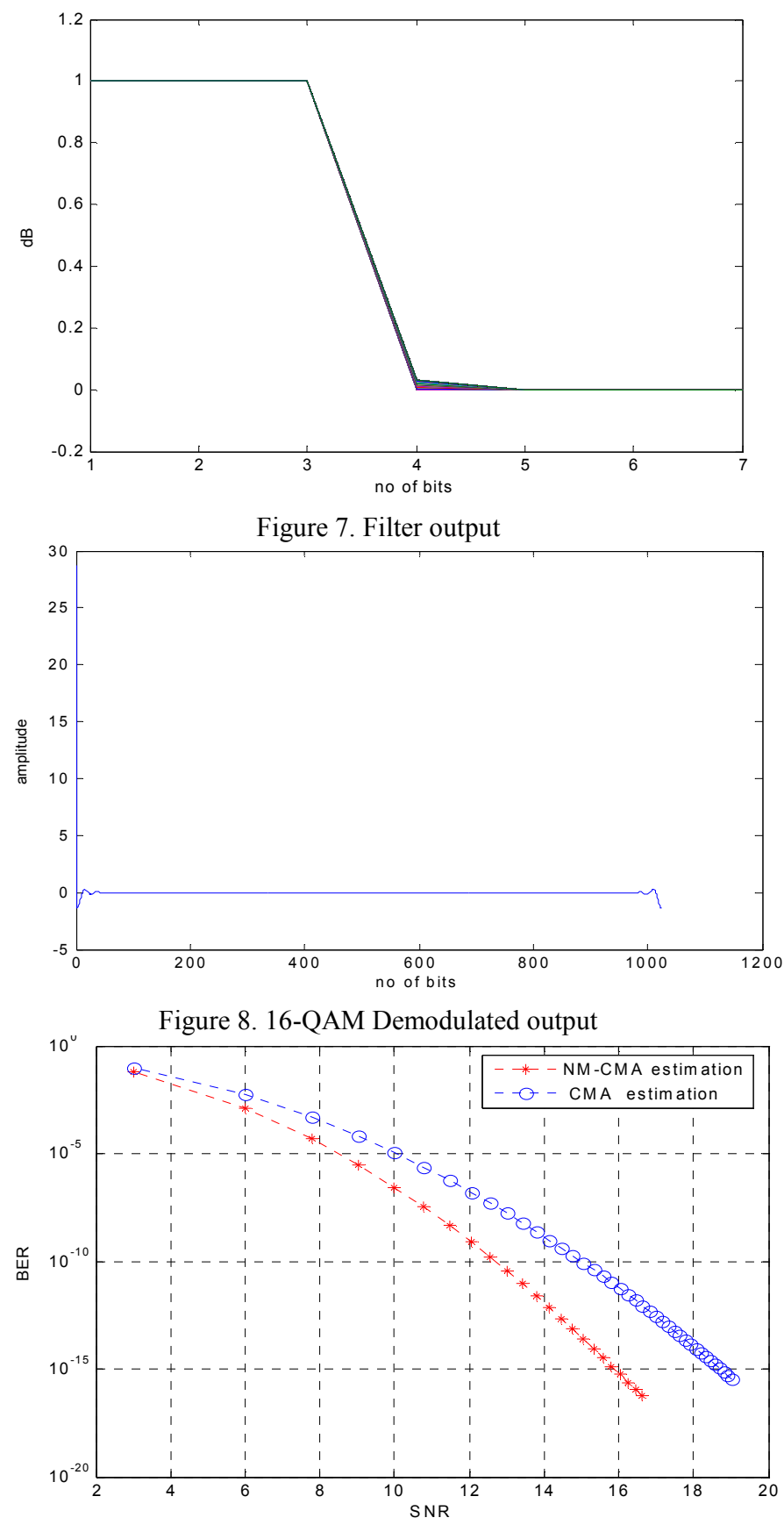

Figure 9. Comparison between BER and SNR of NM-CMA and CMA

\section{CONCLUSIONS}

The CMA technique is proposed in this paper. It has been shown that CMA technique can accomplish blind equalization and carrier phase recovery simultaneously. Taking advantage to handle the non-stability problem and the gradient noise amplification problem. The simulation results show that this technique can certainly achieve higher convergence speed, lower residual intersymbol interference, improved stability and robustness, blind equalization and carrier phase recovery simultaneously especially with heavy-tailed noise, impulsive noise.

\section{REFERENCES}

[1] Y. Sato, "A method of self-recovering equalization for ultilevel amplitude- modulation systems," IEEE Trans. Commun., vol. 23, no. 6, pp.679-682, Jun. 1975.

[2] D. N. Godard, "Self-recovering equalization and carrier tracking in two-dimensional data communications systems," IEEE Trans. Commun., vol. 28, no. 11, pp. 1867-1875, Nov. 1980.

[3] J. R. Treichler and B. G. Agee, "A new approach to multipath correction of constant modulus signals," IEEE Trans. Acoust., Speech, Signal Process., vol. 31, no. 2, pp. 459-472, Apr. 1983.

[4] G. H. Golub and C. F. van Loan, Matrix Computations,3rd ed. Baltimore, MD: Johns Hopkins Univ. Press, 1996.

[5] O. Dabeer and E. Masry, "Convergence analysis of the constant modulus Algorithm Onkar Dabeer, member," IEEE Trans. Inf. Theory, vol. 49, no. 6, pp. 1447-1464, Jun. 2003.

[6] M. T. M. Silva and M. D. Miranda, "Tracking issues of some blind equalization algorithms," IEEE Signal Process. Lett., vol. 11, no. 9 pp. 760-763, Sep. 2004.

[7] C. R. Johnson, Jr., P. Schniter, T. J. Edres, J. D. Behm, D. R. Brown, and R. A. Casas, "Blind equalization using the constant modulus criterion: A review," Proc. IEEE, vol. 86, no. 10, pp. 1927-1950, Oct. 1998.

[8] G. Yan and H. Fan, "A Newton-like algorithm for complex variables with applications in blind equalization," IEEE Trans. Signal Process. vol. 48, no. 2, pp. 553-556, Feb. 2000 . 